\title{
GSTT1 as a prognosticator for recurrence and progression in patients with non-muscle-invasive bladder cancer
}

\author{
Yun-Sok Ha ${ }^{\mathrm{a}}$, Chunri Yan ${ }^{\mathrm{a}, \mathrm{b}}$, Min Su Lym ${ }^{\mathrm{a}, \mathrm{b}}$, Pildu Jeong ${ }^{\mathrm{a}}$, Won Tae Kim ${ }^{\mathrm{a}}$, Yong-June Kim ${ }^{\mathrm{a}, \mathrm{b}}$, \\ Seok-Joong Yun ${ }^{\mathrm{a}}$, Sang-Cheol Lee ${ }^{\mathrm{a}}$, Sung-Kwon Moon ${ }^{\mathrm{c}}$, Yung Hyun Choi ${ }^{\mathrm{d}}$ and Wun-Jae Kim ${ }^{\mathrm{a}} \mathrm{b}, *$ \\ ${ }^{a}$ Department of Urology, College of Medicine, Chungbuk National University, Cheongju, Chungbuk, South Korea \\ ${ }^{\mathrm{b}}$ BK21 Chungbuk Biomedical Science Center, School of Medicine, Chungbuk National University, Cheongju, \\ Chungbuk, South Korea \\ ${ }^{\mathrm{C}}$ Department of Food and Biotechnology, Chungju National University, Chungju, South Korea \\ ${ }^{\mathrm{d}}$ Department of Biochemistry, Dongeui University College of Oriental Medicine and Department of Biomaterial \\ Control (BK21 Program), Dongeui University Graduate School, Busan, South Korea
}

\begin{abstract}
Although polymorphisms in glutathione S-transferase (GST) have been associated with the risk of bladder cancer (BC), few reports provide information about the development of BC. The aim of the present study was to investigate the effect of homozygous glutathione S-transferase- $\mu$ (GSTM1) and glutathione S-transferase- $\phi$ (GSTT1) deletions as prognostic markers in non-muscle-invasive bladder cancer (NMIBC). A total of 241 patients with primary NMIBC were enrolled in this study. GSTM1 and GSTT1 polymorphisms were analyzed by multiplex polymerase chain reaction (PCR) using blood genomic DNA. The results were compared with clinicopathological parameters. The prognostic significance of the GSTs was evaluated by Kaplan-Meier and multivariate Cox regression model. A statistically significant association between genotype and histopathological parameter was not observed. The patients with the GSTT1-positive genotype had significantly reduced recurrence- and progression-free survival than those with the GSTT1-null genotype (log-rank test, $p<0.05$, respectively). Recurrence- and progression-free survival were not related to the GSTM1 genotypes. In multivariate regression analysis, the GSTT1-positive genotype was the independent predictor for recurrence [hazard ratio (HR), 1.631; $p=0.043$ ] and progression (HR, 3.418; $p=0.006)$. These results suggested that the GSTT1 genotype could be a useful prognostic marker for recurrence and progression in NMIBC.
\end{abstract}

Keywords: Urinary bladder neoplasms, polymorphism, biological tumor markers, prognosis

\section{Introduction}

Bladder cancers (BC) are characterized by containing a mixture of heterogeneous cell populations, and numerous factors are likely to be involved in disease recurrence, progression and survival [1]. The prediction of disease recurrence and progression in non-muscle-

\footnotetext{
*Corresponding author: WUN-JAE KIM M.D., Ph.D., Department of Urology, College of medicine, Chungbuk National University 62, Kaeshin-dong, Heungduk-ku, Cheongju, Chungbuk 361-711, Korea. Tel.: +82 43269 6371; Fax: +82 43269 6129; E-mail: wjkim@chungbuk.ac.kr.
}

invasive bladder cancer (NIMBC) is a major clinical issue for urologists. The identification of the aggressive features of the cancer in patients with NMIBC is very important for the adequate management of this disease [2]. Although conventional histopathological variables and diverse biological markers have been proposed to help in the assessment of the prognosis of NMIBC $[3,4]$, useful markers that can predict the accurate clinical course of NMIBCs have not yet been identified.

$\mathrm{BC}$ has been known to be associated with exposure to chemical carcinogens and cigarette smoking [5,6]. Moreover, various enzymes such as $\mathrm{N}$ - 
acetyltransferase 2 (NAT2), glutathione S-transferase$\mu$ (GSTM1), glutathione S-transferase- $\phi$ (GSTT1), and human 8-oxoguanine DNA glycosylase 1 (hOGG1) and others, as well as cytokines and the gene repair system, may be involved in bladder carcinogenesis [7]. The GSTM1-null genotype has attracted attention due to its association with $\mathrm{BC}$, but the risk of $\mathrm{BC}$ in the GSTM1-null type is inconsistent among the different ethnic groups $[6,8,9]$. Reports describing the GSTT1null type as a risk factor in $\mathrm{BC}$ are rare and still controversial [10-12]. Despite numerous single nucleotide polymorphism (SNP) studies in relation to BC, only a few genetic polymorphisms have provided valuable information about the clinicopathologic characteristics of $\mathrm{BC}$ and the prognosis of $\mathrm{BC}$ patients $[13,14]$.

Several environmental factors and the activities of detoxified enzymes have been associated with tumor development in BC, and they could also affect the clinical course of this disease. The putative reasons for this hypothesis are that the patients must be exposed to similar environmental conditions and have the same enzyme activities affected by genetic polymorphisms in even a well-controlled organ-confined cancer. To date, there are no published reports describing the prognostic value of homozygous deletions of GSTM1 and GSTT1 as markers of disease prognosis in primary NMIBC patients with long-term follow-up. The aim of this study is to assess the prognostic value of the GSTM1 and GSTT1 genotype for tumor recurrence and progression in primary NMIBC patients.

\section{Materials and methods}

\subsection{Study population}

A total of 282 patients whose blood samples were eligible to use with transitional cell carcinoma of the urinary bladder were recruited between 1995 and 2008 at our institute. To make the study population more homogeneous, patients with concomitant carcinoma in situ (CIS), short term follow-up period (less than 6 months) and incomplete data collection were excluded. A total of 241 patients with primary NMIBC were included in the study. A blood sample of $5 \mathrm{ml}$ was collected from each patient into $0.1 \mathrm{ml}$ EDTA, frozen in liquid nitrogen, and stored at $-80^{\circ} \mathrm{C}$ until use. A questionnaire structured to obtain a detailed smoking history was administered to each subject by well-trained interviewers. The collection and analysis of samples were approved by the local institutional review board and informed consent was obtained from each subject.

Tumors were staged according to the 2002 TNM classification and the 1973 WHO grading system [4, 15]. A second TUR was performed 2-4 weeks after the initial resection when a bladder cancer specimen did not include proper muscle or when a high-grade was detected [4]. Patients who had multiple tumors, large tumors ( $\geqslant 3 \mathrm{~cm}$ in diameter), or high grade NMIBC received one cycle of intravesical treatment (Bacillus Calmette-Guérin (BCG) or mitomycin-C) [4,16]. Response to treatment was assessed by cystoscopy and urinary cytology. Patients who were free of disease within 3 months after treatment were assessed every 3 months for the first 2 years and every 6 months thereafter $[4,16]$. We defined recurrence as the relapse of primary NMIBC with a lower or equivalent pathologic stage and progression as disease with a higher TNM stage when relapsed.

\subsection{Genotype assays}

Genomic DNA was extracted from human whole blood using a genomic DNA purification kit (Promega, Wisconsin), followed by precipitated with isopropanol samples were washed using $70 \%$ ethanol and the quality of DNA was assessed by agarose gel electrophoresis. Genomic DNA samples were stored at $-20^{\circ} \mathrm{C}$ until use. A multiplex polymerase chain reaction (PCR) method was applied to detect the presence or absence of the GSTM1 and GSTT1 genes in genomic DNA samples, as described previously [17]. In brief, the primers used in the PCR were sense (5'-GAA CTC CCT GAA AAG CTA AAG C- 3') and anti-sense (5'- GTT GGG CTC AAA TAT ACG GTG G - 3') for GSTM1; sense (TTA GCT GAC CTC GTA GCC AT - 3') and anti-sense (5'GAA GTC CTT GGC CTT CAG AA- 3') for GSTT1; and sense (5' - GAA GAG CCA AGG ACA GGT AC3') and anti-sense (5'- CAA CTT CAT CCA CGT TCA CC - 3') for beta-globin ( $\beta$-globin). DNA (200ng) was amplified in a total volume of $20 \mu \mathrm{L}$, containing 10 pmol of each primer, 0.5 unit of Taq polymerase, $2.5 \mathrm{mM}$ dNTP and 10X PCR buffer. Following an initial denaturation step at $94^{\circ}$ for $5 \mathrm{~min}, 40$ cycles of amplification were carried out at $94^{\circ}$ for $60 \mathrm{~s}, 63^{\circ}$ for $60 \mathrm{~s}, 72^{\circ}$ for $60 \mathrm{~s}$ and a final extension step at $72^{\circ}$ for $10 \mathrm{~min}$. The amplified products were electrophoresed on a $2 \%$ agarose gel, and their sizes were $219 \mathrm{bp}$ for GSTM1, 372 bp for GSTT1 and 268 bp for $\beta$-globin. Both positive and negative samples were analyzed in each experiment, and GSTM1 and GSTT1 genotypes were not scored unless the internal reference gene $(\beta$ globin) product was evident. 
Table 1

Clinical and pathological features of non-muscle-invasive bladder cancer

\begin{tabular}{lc}
\hline Variables & $\begin{array}{c}\text { Number of } \\
\text { patients }(\%)\end{array}$ \\
\hline Age-yr (mean) & $62.7 \pm 14.3$ \\
Gender & \\
Male & $197(81.7)$ \\
Female & $44(18.3)$ \\
Stage & \\
Ta & $92(38.2)$ \\
T1 & $149(61.8)$ \\
Grade & \\
G1 & $87(36.1)$ \\
G2 & $121(50.2)$ \\
G3 & $33(13.7)$ \\
Tumor size & \\
$<3$ cm & $144(59.8)$ \\
$\geqslant 3$ cm & $97(40.2)$ \\
Number of tumors & \\
Single & $148(61.4)$ \\
Multiple & $93(38.6)$ \\
Median follow-up - months (Range) & $44.9(6.1-207.8)$ \\
\hline
\end{tabular}

\subsection{Statistical analysis}

Patients were divided into three categories based on the smoking status as follows: smokers (current smokers and patients who quit smoking within 10 years), ex-smokers (smoking had been ended for at least 10 years), and non-smokers (having never smoked) [18]. However, because the number of ex-smokers was relatively small $(N=26,10.8 \%)$ to be analyzed as an independent group, ex-smokers were included in the non-smokers group. The chi-square test was used to analyze the categorical proportions between two groups. The Kaplan-Meier method was used to estimate time to recurrence and progression, and differences were assessed using log-rank statistics. The prognostic value of the GSTM1 and GSTT1 polymorphisms for recurrence and progression in NMIBCs was analyzed with multivariate Cox proportional hazard regression models. Statistical analysis was performed using SPSS 12.0 software (SPSS Inc., Chicago, IL), and a $p$ value of $<$ 0.05 was considered statistically significant.

\section{Results}

\subsection{Baseline characteristics}

Table 1 lists the baseline characteristics of the 241 patients enrolled in this study. The median follow-up period of the primary NMIBC patients was 44.9 months (range, 6.1-207.8). The frequencies of the GSTM1-null and GSTT1-null types were $56.4 \%$ and $63.6 \%$, respectively. Fifty-one percent $(123 / 241)$ of patients were smokers and $49.0 \%(118 / 241)$ non-smokers. Total 76 of 241 patients $(31.5 \%)$ received the repeat TUR for the adequate tumor staging. Intravesical therapy was performed in 98 patients $(40.7 \%)$ after TUR, and 70 patients treated with BCG, and 28 with mitomycin-C. During the follow-up period, $30.7 \%$ (74/241) experienced recurrence, and disease progression was observed in 22 out of $241(9.1 \%)$ patients.

\subsection{Relationship between GSTM1 and GSTT1 genotype, and histopathological parameters according to smoking status}

There were no significant associations between smoking status and histopathological parameters such as tumor stage and grade. After stratification by smoking status, no association between GSTM1, GSTT1 genotypes and histopathological features of NMIBC were found (Table 2).

\subsection{Genotype of GSTM1 and GSTT1, and prognosis for NMIBC}

Kaplan-Meier estimates revealed significant differences in time to tumor recurrence and progression according to the GSTT1 genotype. The patients with the GSTT1-positive genotype had significantly reduced recurrence- and progression-free survival than those with the GSTT1-null genotype (log-rank test, $p=0.034$ and $p=0.001$, respectively, Fig. $1 \mathrm{~A}$ and B). However, recurrence- and progression-free survival were not related to the GSTM1 genotypes.

Univariate Cox regression analysis revealed that $\mathrm{T} 1$ stage [hazard ration (HR), 1.806; $95 \%$ confidence interval (CI), 1.072-3.042; $p=0.026]$ and GSTT1-positive genotype [HR, 1.646; 95\% CI, 1.035-2.617; $p=$ 0.035] showed a significant impact on recurrence of bladder cancer. On multivariate analysis, however, the GSTT1-positive genotype [HR, 1.631; 95\% CI, 1.015$2.621 ; p=0.043$ ] was the only independent predictor of bladder tumor recurrence (Table 3). Moreover, Univariate analysis showed that elder age [HR, 1.052; 95\% CI, 1.014-1.091; $p=0.007]$, bigger sized tumor [HR, 2.821; 95\% CI, 1.183-6.727; $p=0.019$ ], grade G3 [HR, 6.449; 95\% CI, 1.879-22.133; $p=0.003$ ] and GSTT1-positive genotype [HR, 3.733; 95\% CI, 1.5798.826; $p=0.003$ ] were the risk factors for progression of bladder cancer. However, the GSTT1-positive genotype (HR, 3.418; 95\% CI, 1.420-8.227; $p=0.006$ ) was the only independent predictor of bladder cancer progression on multivariate analysis (Table 4). 
Table 2

Relationship between GSTM1 and GSTT1 genotypes, and stage and grade stratified by smoking status in non-muscle-invasive bladder cancer

\begin{tabular}{|c|c|c|c|c|c|c|}
\hline \multirow[t]{2}{*}{ Variables } & \multicolumn{3}{|c|}{ GSTM1 } & \multicolumn{2}{|c|}{ GSTT1 } & \multirow[b]{2}{*}{$p$} \\
\hline & Null $(\%)$ & Positive (\%) & $p$ & Null (\%) & Positive (\%) & \\
\hline \multicolumn{7}{|c|}{ Non-smokers $(N=118)$} \\
\hline Stage & & & 0.632 & & & 0.571 \\
\hline $\mathrm{Ta}$ & $23(51.1)$ & $22(48.9)$ & & $26(57.8)$ & $19(42.2)$ & \\
\hline $\mathrm{T} 1$ & $34(46.6)$ & $39(53.4)$ & & $46(63.0)$ & $27(37.0)$ & \\
\hline Grade & & & 0.369 & & & 0.831 \\
\hline G1 & $18(42.9)$ & $24(57.1)$ & & $26(61.9)$ & $16(38.1)$ & \\
\hline $\mathrm{G} 2$ & $25(44.6)$ & $31(55.4)$ & & $35(62.5)$ & $21(37.5)$ & \\
\hline G3 & $14(70.0)$ & $6(30.0)$ & & $11(55.0)$ & $9(45.0)$ & \\
\hline \multicolumn{7}{|c|}{ Smokers $(N=123)$} \\
\hline Stage & & & 0.483 & & & 0.233 \\
\hline $\mathrm{Ta}$ & $32(68.1)$ & $15(31.9)$ & & $34(72.3)$ & $13(27.7)$ & \\
\hline $\mathrm{T} 1$ & $47(61.8)$ & $29(38.2)$ & & $47(61.8)$ & $29(38.2)$ & \\
\hline Grade & & & 0.434 & & & 0.845 \\
\hline G1 & $27(60.0)$ & $18(40.0)$ & & $31(68.9)$ & $14(31.1)$ & \\
\hline G2 & $45(69.2)$ & $20(30.8)$ & & $42(64.6)$ & $23(35.4)$ & \\
\hline G3 & $7(53.8)$ & $6(46.2)$ & & 8 (61.5) & $5(38.5)$ & \\
\hline
\end{tabular}

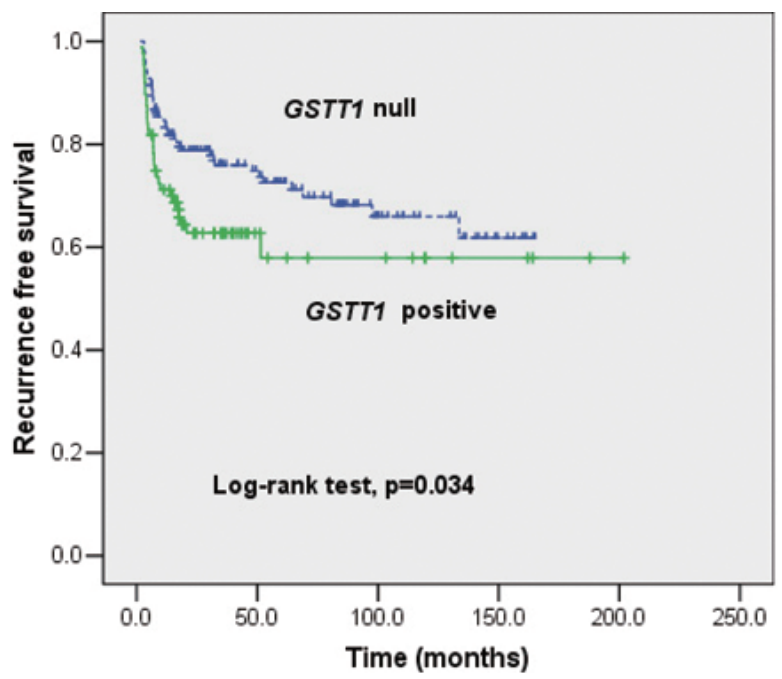

(a)

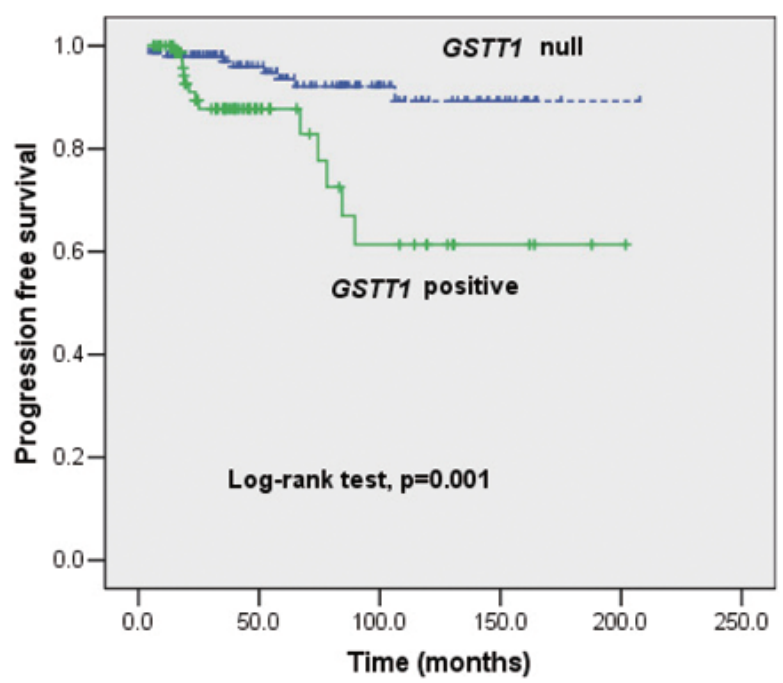

(b)

Fig. 1. Kaplan-Meier estimate curves predict the probability of recurrence and progression according to the GSTT1 genotypes. Recurrence (A) and progression $(\mathrm{B})$ in patients with non-muscle-invasive bladder cancer.

\section{Discussion}

The activities of specific enzymes can change with genotype. Specific enzymes that are known to be important in carcinogenesis can also play a critical role in disease recurrence and progression after initial treatment. The results of the present study demonstrated that the GSTT1 genotype was a strong indicator for predicting recurrence and progression in patients with primary NMIBC. The majority of published studies addressing GSTT1 genotype have been focused on the development of $\mathrm{BC}$ as a risk or as protective factor $[10,11$,
18-22]. However, studies of the associations between GSTT1 genotype and the development of BC have reported controversial results. We previously reported that the GSTT1-null genotype was significantly associated with the development of BC, and that a GSTT1-null genotype was not a risk factor but a protective factor of $\mathrm{BC}$ [11]. The findings of the present study support our previous data.

Although the GSTM1-null genotype has been associated with increased risk of $\mathrm{BC}$ in previous reports [23-25], the current study revealed that GSTM1 genotype was not associated with histopathologic pa- 
Table 3

Univariate and multivariate Cox regression analysis for prediction of recurrence in non-muscle-invasive bladder cancer

\begin{tabular}{lccccc}
\hline Variables & \multicolumn{2}{c}{ Univariate analysis } & & \multicolumn{2}{c}{ Multivariate analysis } \\
\cline { 2 - 3 } \cline { 5 - 6 } & HR $(95 \%$ CI $)$ & $p$ & & HR $(95 \%$ CI $)$ & $p$ \\
\hline Age & $1.010(0.993-1.027)$ & 0.240 & & $1.004(0.986-1.023)$ & 0.639 \\
Sex (male vs. female) & $0.720(0.369-1.402)$ & 0.334 & & $0.719(0.363-1.426)$ & 0.345 \\
Smoking status (non smokers vs. smokers) & $1.126(0.711-1.782)$ & 0.613 & & $1.114(0.692-1.791)$ & 0.657 \\
Number of tumors (single vs. multiple) & $1.538(0.972-2.433)$ & 0.066 & & $1.369(0.838-2.235)$ & 0.209 \\
Tumor size (<3 cm vs. $\geqslant 3)$ & $1.363(0.864-2.152)$ & 0.183 & & $1.081(0.659-1.772)$ & 0.757 \\
Stage (Ta vs. T1) & $1.806(1.072-3.042)$ & 0.026 & & $1.559(0.814-2.986)$ & 0.180 \\
Grade & & & & & \\
$\quad$ G1 & 1 & & & & - \\
G2 & $1.401(0.826-2.376)$ & 0.211 & & $0.950(0.499-1.807)$ & 0.875 \\
G3 & $1.892(0.946-3.783)$ & 0.072 & & $1.206(0.522-2.782)$ & 0.661 \\
GSTT1 (null vs. positive) & $1.646(1.035-2.617)$ & 0.035 & & $1.631(1.015-2.621)$ & 0.043 \\
\hline
\end{tabular}

Table 4

Univariate and multivariate Cox regression analysis for prediction of progression in non-muscle-invasive bladder cancer

\begin{tabular}{lccccc}
\hline Variables & \multicolumn{2}{c}{ Univariate analysis } & & \multicolumn{2}{c}{ Multivariate analysis } \\
\cline { 2 - 3 } \cline { 5 - 6 } & HR $(95 \%$ CI $)$ & $p$ & & HR $(95 \%$ CI $)$ & $p$ \\
\hline Age & $1.052(1.014-1.091)$ & 0.007 & & $1.040(1.000-1.082)$ & 0.052 \\
Sex (male vs. female) & $0.837(0.247-2.830)$ & 0.774 & & $0.838(0.234-2.994)$ & 0.785 \\
Smoking status (non smokers vs. smokers) & $1.089(0.463-2.558)$ & 0.845 & & $1.402(0.562-3.499)$ & 0.469 \\
Number of tumors (single vs. multiple) & $1.349(0.573-3.176)$ & 0.493 & & $1.017(0.416-2.486)$ & 0.970 \\
Tumor size (<3 cm vs. $\geqslant 3)$ & $2.821(1.183-6.727)$ & 0.019 & & $1.960(0.756-5.077)$ & 0.166 \\
Stage (Ta vs. T1) & $2.331(0.789-6.893)$ & 0.126 & & $0.937(0.224-3.926)$ & 0.929 \\
Grade & & & & & \\
G1 & 1 & & & \\
G2 & $1.969(0.627-6.186)$ & 0.246 & & $1.304(0.315-5.393)$ & 0.714 \\
G3 & $6.449(1.879-22.133)$ & 0.003 & & $3.091(0.628-15.220)$ & 0.165 \\
GSTT1 (null vs. positive) & $3.733(1.579-8.826)$ & 0.003 & & $3.418(1.420-8.227)$ & 0.006 \\
\hline
\end{tabular}

rameters or with the prognosis for NMIBC. After stratification by smoking status, no associations were detected between GSTMI genotype and clinical outcomes. Cigarette smoking has been associated with the development of BC in many epidemiologic studies [6,8]. Some studies reported that genetic polymorphism relevant to BC could only be detected in smokers $[6,14]$. Since GSTs are related to the metabolism of hazardous chemicals or cigarette smoking, those previous reports seemed to have the causal relationship [6,14]. However, the results of other studies indicated that smoking was not a significant risk factor for BC $[9,26]$. These discrepancies might reflect differences among ethnic groups or heterogeneous study populations. The current data do not represent results of a case-control study designed to identify factors associating smoking status and the development of BC. In the present study, smoking status related to GSTM1 genotype was not associated with recurrence and progression in NMIBC patients.

Few studies have analyzed the prognostic value of genetic polymorphisms for different types of cancers including BC. A study by Song et al. reported that the NAT2 slow-acetylator genotype presented a significant risk for high grade and stage in BC [14]. Castillejo et al. found that the transforming growth factor-beta receptors $(T G F B R 1)$ rs868 allele was strongly and independently associated with disease-specific mortality among patients with muscle invasive bladder cancer (MIBC) [27]. In their study, other polymorphic variants analyzed were not found to be associated with an increased risk of $\mathrm{BC}$, but the TGFBR1 rs868 allele was identified as a prognostic marker. Among patients with non-small-cell lung cancer, the functional $\mathrm{Y} / \mathrm{X}$ polymorphism of the innate-immunity gene mannose-binding lectin $(\mathrm{M} B L) 2$, as well as $M B L 2$ haplotypes and diplotypes, was found to be associated with lung cancer-specific survival among caucasian patients [28]. In another study including patients diagnosed with breast cancer, genetic variations in signalinduced proliferation-associated gene 1 (SIPAI) were not related to breast cancer metastasis or survival [29]. In the current study, GSTT1 genotype was found to be a strong prognostic factor for tumor recurrence 
(HR, 1.631) and progression (HR, 3.418) in primary NMIBC. The results obtained in the present study are strengthened by certain aspects of the study design, such as the inclusion of a relatively large-scale population that consisted only of patients with NMIBC and the long-term follow-up (median follow-up, 44.9 months).

GSTs have been studied for their role in detoxification through the conjugation of a wide variety of potentially toxic and carcinogenic electrophiles with glutathione [30]. The exact mechanisms by which GSTT1 affects the progression of $\mathrm{BC}$, however, remain to be elucidated. To further investigate this mechanism, our group is currently performing tissue genotype analysis and evaluating the expression levels of GSTM1 and GSTT1 in BC tissues.

Although various diagnostic markers for BC development, recurrence, and progression have been reported, adequate indicators able to predict the prognosis of most BCs have not been found yet. The present study shows that the GSTT1 genotype could be a better prognostic indicator for the progression of NMIBC than previously described markers, mostly due to the method of blood sampling, which was easier to carry out than examination based on tissue samples. The use of the GSTT1 genotype as a prognostic indicator, independent of traditional pathologic prognostic parameters, could potentially enable the separation of heterogeneous NMIBC patients into good and poor prognostic groups, and may enable individualized evaluation and design of treatment strategies.

In conclusion, the present study showed that the GSTT1 genotype may play an important role in the prognosis of NMIBC in the clinical setting.

\section{Acknowledgements}

This research was supported by the Basic Science Research Program through the National Research Foundation of Korea (NRF) funded by the Ministry of Education, Science and Technology (2010-0001730).

\section{References}

[1] W.J. Kim, S. Park and Y.J. Kim, Biomarkers in bladder cancer: present status and perspectives, Biomark Insights 2 (2007), 95-105.

[2] L.S. Borden Jr, P.E. Clark and M.C. Hall, Bladder cancer, Curr Opin Oncol 15 (2003), 227-233.

[3] T.T. Wu, J.H. Chen, Y.H. Lee et al., The role of bcl-2, p53, and ki-67 index in predicting tumor recurrence for low grade superficial transitional cell bladder carcinoma, J Urol 163 (2000), $758-760$.
[4] M. Babjuk, W. Oosterlinck, R. Sylvester et al., EAU guidelines on non-muscle-invasive urothelial carcinoma of the bladder, Eur Urol 54 (2008), 303-314.

[5] G.R. Howe, J.D. Burch, A.B. Miller et al., Tobacco use, occupation, coffee, various nutrients, and bladder cancer, J Natl Cancer Inst 64 (1980), 701-713.

[6] D.A. Bell, J.A. Taylor, D.F. Paulson et al., Genetic risk and carcinogen exposure: a common inherited defect of the carcinogen-metabolism gene glutathione S-transferase M1 (GSTM1) that increases susceptibility to bladder cancer, J Natl Cancer Inst 85 (1993), 1159-1164.

[7] Y. Hirao, W.J. Kim and K. Fujimoto, Environmental factors promoting bladder cancer, Curr Opin Urol 19 (2009), 494499.

[8] T. Katoh, H. Inatomi, H. Kim et al., Effects of glutathione S-transferase (GST) M1 and GSTT1 genotypes on urothelial cancer risk, Cancer Lett 132 (1998), 147-152.

[9] W.J. Kim, H.L. Lee, S.C. Lee et al., Polymorphisms of Nacetyltransferase 2, glutathione S-transferase mu and theta genes as risk factors of bladder cancer in relation to asthma and tuberculosis, J Urol 164 (2000), 209-213.

[10] J. Brockmoller, I. Cascorbi, R. Kerb et al., Combined analysis of inherited polymorphisms in arylamine $\mathrm{N}$-acetyltransferase 2, glutathione S-transferases M1 and T1, microsomal epoxide hydrolase, and cytochrome P450 enzymes as modulators of bladder cancer risk, Cancer Res 56 (1996), 3915-3925.

[11] W.J. Kim, H. Kim, C.H. Kim et al., GSTT1-null genotype is a protective factor against bladder cancer, Urology 60 (2002), 913-918.

[12] M. McGrath, D. Michaud and I. De Vivo, Polymorphisms in GSTT1, GSTM1, NAT1 and NAT2 genes and bladder cancer risk in men and women, BMC Cancer 6 (2006), 239.

[13] E.J. Kim, P. Jeong, C. Quan et al., Genotypes of TNF-alpha, VEGF, hOGG1, GSTM1, and GSTT1: useful determinants for clinical outcome of bladder cancer, Urology 65 (2005), $70-75$.

[14] D.K. Song, D.L. Xing, L.R. Zhang et al., Association of NAT2, GSTM1, GSTT1, CYP2A6, and CYP2A13 gene polymorphisms with susceptibility and clinicopathologic characteristics of bladder cancer in Central China, Cancer Detect Prev 32 (2009), 416-423.

[15] F.L. Greene, The American Joint Committee on Cancer: updating the strategies in cancer staging, Bull Am Coll Surg 87 (2002), 13-15.

[16] M.C. Hall, S.S. Chang, G. Dalbagni et al., Guideline for the management of non muscle invasive bladder cancer (stages Ta, T1, and Tis): 2007 update, J Urol 178 (2007), 2314-2330.

[17] H. Chen, D.P. Sandler, J.A. Taylor et al., Increased risk for myelodysplastic syndromes in individuals with glutathione transferase theta 1 (GSTT1) gene defect, Lancet 347 (1996), 295-297.

[18] M. Kempkes, K. Golka, S. Reich et al., Glutathione Stransferase GSTM1 and GSTT1 null genotypes as potential risk factors for urothelial cancer of the bladder, Arch Toxicol 71 (1996), 123-126.

[19] R.J. Hung, P. Boffetta, P. Brennan et al., GST, NAT, SULT1A1, CYP1B1 genetic polymorphisms, interactions with environmental exposures and bladder cancer risk in a high-risk population, Int J Cancer 110 (2004), 598-604.

[20] J. Salagovic, I. Kalina, V. Habalova et al., The role of human glutathione S-transferases M1 and T1 in individual susceptibility to bladder cancer, Physiol Res 48 (1999), 465-471.

[21] X. Giannakopoulos, K. Charalabopoulos, D. Baltogiannis et al., The role of N-acetyltransferase- 2 and glutathione S- 
transferase on the risk and aggressiveness of bladder cancer, Anticancer Res 22 (2002), 3801-3804.

[22] S.J. Lee, S.H. Cho, S.K. Park et al., Combined effect of glutathione S-transferase M1 and T1 genotypes on bladder cancer risk, Cancer Lett 177 (2002), 173-179.

[23] M. Garcia-Closas, N. Malats, D. Silverman et al., NAT2 slow acetylation, GSTM1 null genotype, and risk of bladder cancer: results from the Spanish Bladder Cancer Study and metaanalyses, Lancet 366 (2005), 649-659.

[24] L.E. Johns and R.S. Houlston, Glutathione S-transferase mu1 (GSTM1) status and bladder cancer risk: a meta-analysis, Mutagenesis 15 (2000), 399-404.

[25] L.S. Engel, E. Taioli, R. Pfeiffer et al., Pooled analysis and meta-analysis of glutathione S-transferase M1 and bladder cancer: a HuGE review, Am J Epidemiol 156 (2002), 95-109.

[26] K. Golka, T. Schmidt, T. Seidel et al., The influence of poly- morphisms of glutathione S-transferases M1 and M3 on the development of human urothelial cancer, $J$ Toxicol Environ Health A 71 (2008), 881-886.

[27] A. Castillejo, N. Rothman, C. Murta-Nascimento et al., TGFB1 and TGFBR1 polymorphic variants in relationship to bladder cancer risk and prognosis, Int J Cancer 124 (2009), 608-613.

[28] S.R. Pine, L.E. Mechanic, S. Ambs et al., Lung cancer survival and functional polymorphisms in MBL2, an innate-immunity gene, J Natl Cancer Inst 99 (2007), 1401-1409.

[29] M.M. Gaudet, K. Hunter, P. Pharoah et al., Genetic variation in SIPA1 in relation to breast cancer risk and survival after breast cancer diagnosis, Int J Cancer 124 (2009), 1716-1720.

[30] R.C. Strange, P.W. Jones and A.A. Fryer, Glutathione Stransferase: genetics and role in toxicology, Toxicol Lett 112113 (2000), 357-363. 


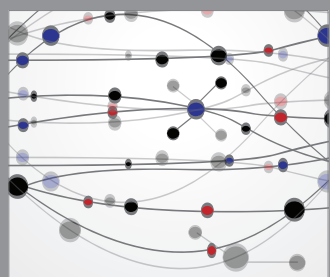

The Scientific World Journal
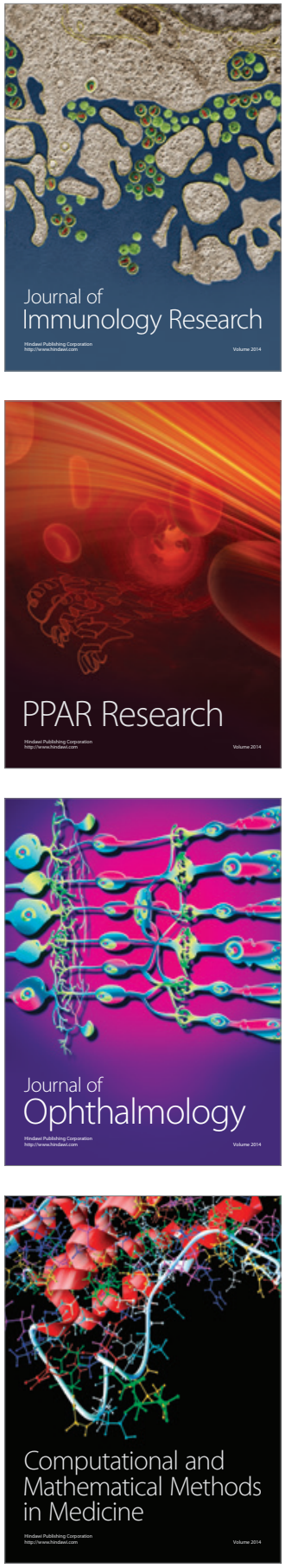

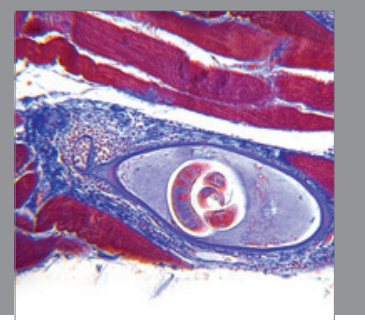

Gastroenterology

Research and Practice
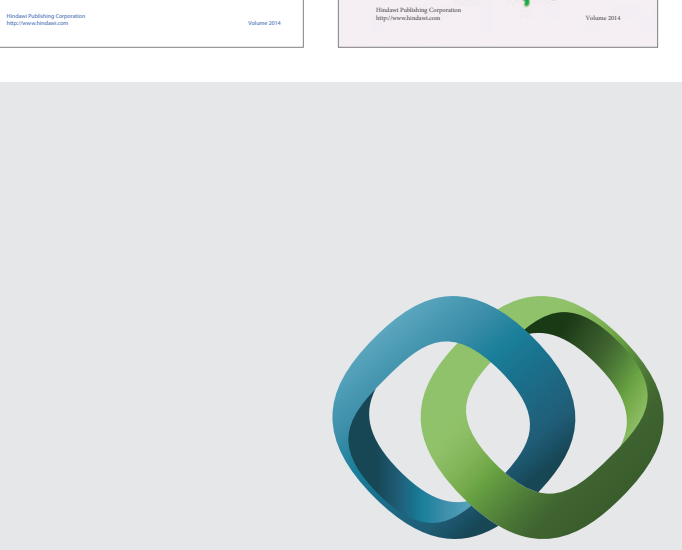

\section{Hindawi}

Submit your manuscripts at

http://www.hindawi.com
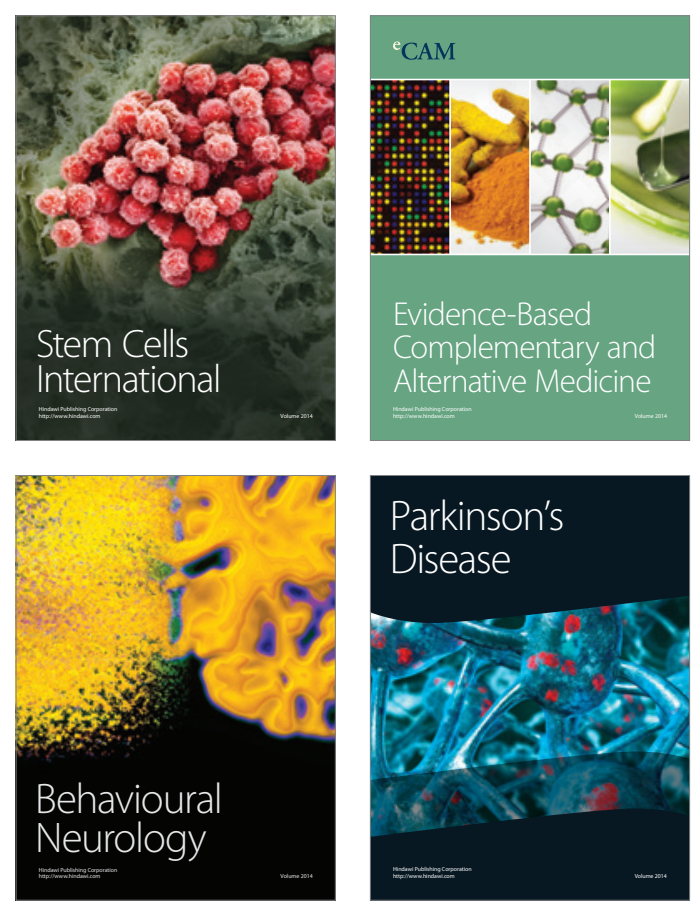

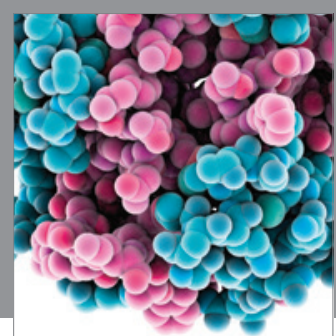

Journal of
Diabetes Research

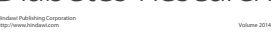

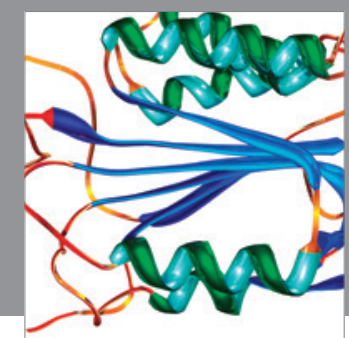

Disease Markers
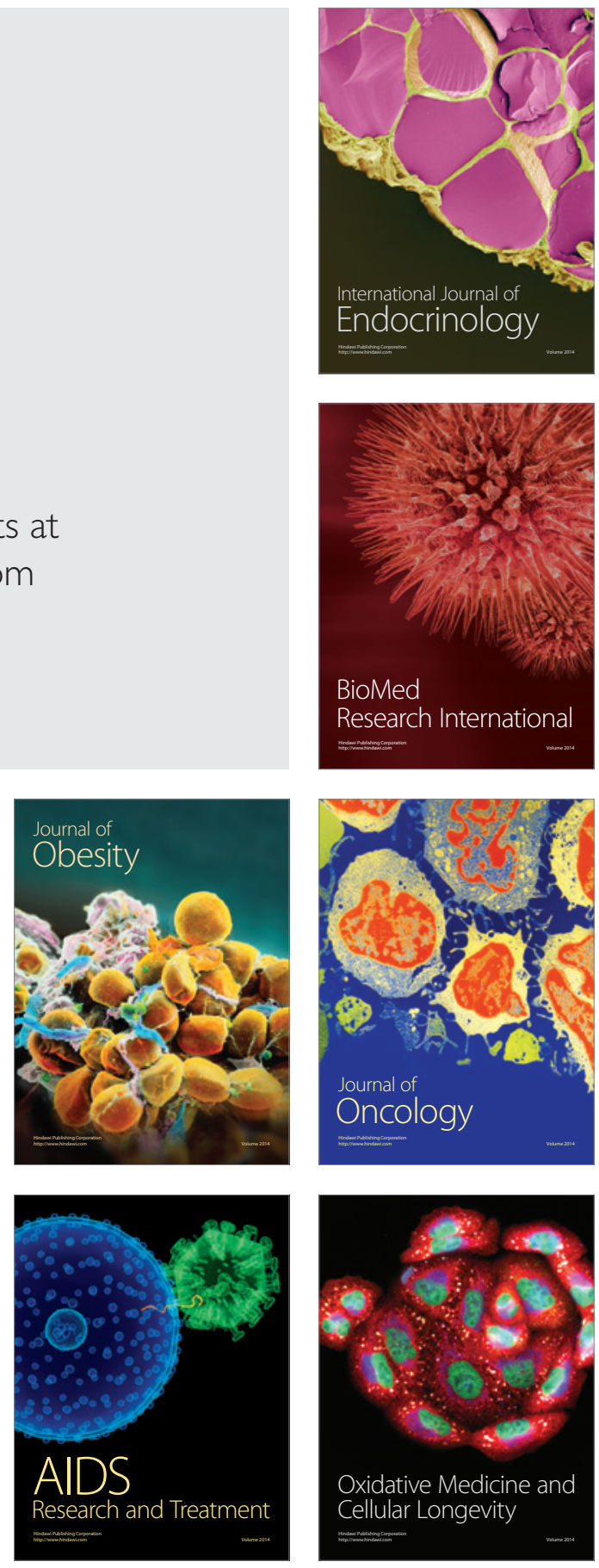